\title{
Orthognathic surgery of temporomandibular disorders
}

\author{
Jong-Ki Huh, DDS, MSD, PhD \\ Section Editor of JKAOMS \\ Department of Oral and Maxillofacial Surgery, Gangnam Severance Hospital, Yonsei University College of Dentistry, Seoul, Korea
}

The treatment of temporomandibular disorders (TMD) remains a difficult task in the field of oral and maxillofacial surgery. The surgical field associated with TMD, especially temporomandibular joint (TMJ) disorders (TMJD), may include the surgical treatment of joint disorders, joint diseases, trauma, or facial deformities caused by the TMD.

Patients with skeletal class III malocclusion and mandibular prognathism rarely exhibit TMD; however, patients with skeletal class II malocclusion and mandibular retrognathism are often associated with TMD ${ }^{1}$. In people of European and North American descent, the mandibular bone is less developed, and the motive for orthognathic surgery is different from those of Southeast Asian descent, including Koreans, because many of these patients have skeletal class II malocclusion or mandibular retrognathism ${ }^{2,3}$. When the mandible is less developed in Koreans, however, oftentimes the mandible grows less or shows degenerative changes due to other causes rather than the original skeleton. Mandibular hypoplasia, condylar resorption due to osteoarthritis, regressive remodeling of the mandibular condyle and ramus, and deformities such as hemifacial microsomia are some potential causes. In addition, in the case of fibrous/bony ankylosis of the TMJ due to trauma during the growth period and resulting facial asymmetry due to growth delay of the ipsilateral side, additional surgeries, as well as TMJ surgery and orthognathic surgery, are performed either simultaneously or at intervals depending on the growth period.

\footnotetext{
Jong-Ki Huh

Department of Oral and Maxillofacial Surgery, Gangnam Severance Hospital, Yonsei University College of Dentistry, 211 Eonju-ro, Gangnamgu, Seoul 06273, Korea

TEL: +82-2-2019-4560

E-mail:omshuh@yuhs.ac

ORCID: https://orcid.org/0000-0002-7381-3972

(c) This is an open-access article distributed under the terms of the Creative Commons Attribution Non-Commercial License (http://creativecommons.org/ licenses/by-nc/4.0/), which permits unrestricted non-commercial use, distribution, and reproduction in any medium, provided the original work is properly cited.

Copyright (C) 2021 The Korean Association of Oral and Maxillofacial Surgeons. All rights reserved.
}

In patients with TMD and severely reduced condyle shape or volume, orthognathic surgery repositions the mandible anteriorly. Oftentimes, it is difficult to locate the mandibular condyle that fits well in the glenoid fossa during surgery, and although it was previously thought that the condylar position was well established at the time of the operation, the position of condyle might change as the muscle is reactivated after surgery. This situation is caused by structural disharmony between the mandibular condyle and the glenoid fossa, and it occurs rarely in patients with skeletal class III malocclusion or mandibular prognathism that have a large mandibular condyle or are structurally well matched. Therefore, when formulating a surgical plan for patients with a small mandibular condyle, surgeons will be helped by checking the following: whether the size of the mandibular condyle is in harmony with the size of the glenoid fossa, what position the patient's mandible is most comfortable with/without considering occlusion, and the severity of the discrepancy between the maximum intercuspation (centric occlusion [CO]) and centric relation (CR). Therefore, even if the mandibular condyle is small, mandibular hypoplasia structurally harmonious with the glenoid fossa is less difficult to position the mandibular condyle during surgery, but if the structural difference between the mandibular condyle and the glenoid fossa is large, such as regressive remodeling of the condylar process or reduced condylar head volume by osteoarthritis or condylar resorption, it is necessary to position the mandibular condyle well during surgery.

If the mandibular condyle is smaller due to a TMD, an evaluation of the TMD should be made before and after orthognathic surgery. Cases in which the mandibular condyle continues to be resorbed and mandibular retrusion occurs despite a successful surgery are difficult for both the patient and the surgeon. Therefore, TMD evaluation is important prior to surgery, whether the patient's TMJ condition is well adapted and exhibits normal movement patterns, or whether there is any discomfort or pain in the TMJ due to poor adap- 
tation, or whether there is a limitation in the range of motion of the mandible. If there are signs and symptoms of TMD, patients should receive evaluation and treatment for the TMD before surgery. Severe TMJD is sometimes associated with systemic diseases, so consultation with related departments such as rheumatology, endocrinology, rehabilitation medicine, and family medicine is recommended before surgery. Of course, cases which demonstrate no TMJ problem in the pre-operative evaluation can easily undergo orthognathic surgery, but some patients complain of symptoms due to TMD six months, one year, or many years following surgery ${ }^{4}$ Patients who show a pattern of TMD recurrence should consult a TMD treatment specialist to determine whether the cause is due to orthognathic surgery or other causes.

In cases of severe condylar resorption showing few remained condylar process, severe mandibular retrusion, anterior open bite, or severe CO-CR discrepancy, it is not only difficult to locate the condylar head during orthognathic surgery, but it is also expected that recurrence of condylar resorption will proceed after surgery. The surgeon should consider orthognathic surgery combined with alloplastic total joint replacement in these cases 5 . In addition, many of these patients have sleep disorders due to a narrow airway space by mandibular retrusion, so sleep evaluation through polysomnography is necessary before surgery.

Since symptom improvement is sometimes difficult in the treatment of severe TMJD, surgeons must carefully check and treat various factors before, during, and after orthognathic surgery for patients with TMJD.

\section{Conflict of Interest}

No potential conflict of interest relevant to this article was reported.

\section{References}

1. Manfredini D, Segù M, Arveda N, Lombardo L, Siciliani G, Alessandro Rossi, et al. Temporomandibular joint disorders in patients with different facial morphology. A systematic review of the literature. J Oral Maxillofac Surg 2016;74:29-46. https://doi. org/10.1016/j.joms.2015.07.006

2. Baik HS, Han HK, Kim DJ, Proffit WR. Cephalometric characteristics of Korean Class III surgical patients and their relationship to plans for surgical treatment. Int J Adult Orthodon Orthognath Surg 2000;15:119-28.

3. Proffit WR, Jackson TH, Turvey TA. Changes in the pattern of patients receiving surgical-orthodontic treatment. Am J Orthod Dentofacial Orthop 2013;143:793-8. https://doi.org/10.1016/ j.ajodo.2013.01.014

4. Al-Moraissi EA, Wolford LM, Perez D, Laskin DM, Ellis E 3rd. Does orthognathic surgery cause or cure temporomandibular disorders? A systematic review and meta-analysis. J Oral Maxillofac Surg 2017;75:1835-47. https://doi.org/10.1016/j.joms.2017.03.029

5. Chung CJ, Choi YJ, Kim IS, Huh JK, Kim HG, Kim KH. Total alloplastic temporomandibular joint reconstruction combined with orthodontic treatment in a patient with idiopathic condylar resorption. Am J Orthod Dentofacial Orthop 2011;140:404-17. https:// doi.org/10.1016/j.ajodo.2009.12.037

How to cite this article: Huh JK. Orthognathic surgery of temporomandibular disorders. J Korean Assoc Oral Maxillofac Surg 2021;47:63-64. https://doi.org/10.5125/jkaoms.2021.47.2.63 